La Revue

des Droits

de l'Homme

\section{La Revue des droits de l'homme}

Revue du Centre de recherches et d'études sur les droits fondamentaux

$10 \mid 2016$

Revue des droits de l'homme - $\mathrm{N}^{\circ} 10$

\title{
Les lanceurs d'alerte dans les pays en transition démocratique : les enseignements tirés de l'expérience tunisienne.
}

Souheil Kaddour

\section{OpenEdition}

Édition électronique

URL : http://journals.openedition.org/revdh/2435

DOI : $10.4000 /$ revdh.2435

ISSN : 2264-119X

Éditeur

Centre de recherches et d'études sur les droits fondamentaux

Référence électronique

Souheil Kaddour, «Les lanceurs d'alerte dans les pays en transition démocratique : les enseignements tirés de l'expérience tunisienne. », La Revue des droits de l'homme [En ligne], 10 | 2016, mis en ligne le 07 juillet 2016, consulté le 25 juillet 2020. URL : http://journals.openedition.org/revdh/2435 ; DOI : https://doi.org/10.4000/revdh.2435

Ce document a été généré automatiquement le 25 juillet 2020.

Tous droits réservés 


\title{
Les lanceurs d'alerte dans les pays en transition démocratique : les enseignements tirés de l'expérience tunisienne.
}

\author{
Souheil Kaddour
}

\section{NOTE DE L'AUTEUR}

Cette étude a été présentée au colloque international sur « Les lanceurs d'alerte et les droits de l'Homme », organisé par l'université Paris-Sorbonne et l'université ParisNanterre, à Paris, en France, les 8 et 9 avril 2015.

\section{Introduction}

Poète icône de la Tunisie au XXème siècle, Aboulkacem Al-Chebbi nous légua deux vers immortels :

« Lorsqu'un jour le peuple voudra vivre,

Le destin l'exaucera infailliblement,

La nuit se dissipera certainement,

Et le carcan se brisera immanquablement ».

Du 17 décembre 2010 au 14 janvier 2011, date officielle de la Révolution de la liberté et de la dignité en Tunisie, le carcan s'est brisé, les esprits se sont révoltés, les langues se sont déliées, les murs de la terreur ont été détruits, une capacité à des actions pacifiques non violentes a été découverte et un nouveau narratif national a commencé à émerger : un narratif de dignité, d'espoir, de reprise de pouvoir et de parole ${ }^{1}$.

3 La parole démocratique qui, justement, lorsqu'elle est reprise par des "lanceurs d'alerte» («mouballigh» en langue arabe, «whistleblower» en langue anglaise) 
évoque le signalement ou la révélation d'informations, de bonne foi, librement et dans l'intérêt général, des manquements graves à la loi ou des risques graves menaçant des intérêts publics ou privés².

4 Cette parole, enfin libre, et ce porteur de parole, supposé libéré, commandent la protection pour que l'autoritarisme, l'oppression, l'omerta, la violation de la loi ne se reproduisent plus. C'est une dette de la Révolution tunisienne envers les lanceurs d'alerte : TuniLeaks, les documents dévoilés par Wikileaks sur la Tunisie en novembre 2010 ne présageaient-ils pas les étincelles de cette Révolution ${ }^{3}$ ?

On s'attendrait donc à ce que la transition démocratique en Tunisie ${ }^{4}$ réhabilite, en droit et en fait, ces "contrepoids parmi les contre-pouvoirs " ${ }^{5}$. Surtout qu'on est dans le temps d'émergence internationale des législations protectrices des lanceurs d'alerte ${ }^{6}$.

Notre dessein dans cette étude est de mettre en exergue, sous l'angle de la transition démocratique la spécificité et l'originalité du rôle des lanceurs d'alerte ainsi que la précarité et l'incomplétude de leur statut dans le processus de transition démocratique en Tunisie.

7 Signalons de prime abord que la «spécificité » de l'expérience tunisienne ne contredit en rien « l'universalité » des dilemmes posés par la problématique de l'alerte.

8 Au plan de l'universalité des questions relatives aux lanceurs d'alerte, on retrouve pratiquement les mêmes interrogations et contradictions : l'alerte éthique constitue-telle un droit ou une obligation? L'alerte éthique est-elle un droit subjectif? Une déclinaison du droit à la liberté d'expression? Un éventuel droit de résistance à l'oppression? Comment concilier des exigences démocratiques opposées : protection des tiers et du débat public contre les assertions diffamatoires et nécessité de libérer la parole des lanceurs d'alerte? Comment concilier alerte éthique et respect du devoir hiérarchique, protection du secret professionnel et obligation de discrétion professionnelle ? Comment concilier les impératifs de la liberté de la presse, du droit à l'information, de la liberté d'expression et de communication avec les exigences du secret de l'instruction, de la lutte contre le terrorisme, du droit au respect de la vie privée, de la protection des données personnelles, de la présomption d'innocence?

9 Au plan de la spécificité de l'expérience tunisienne, on relève certainement cet engouement des citoyens-lanceurs d'alerte à la dénonciation des abus, principalement des cas de corruption, via les réseaux sociaux pour diverses raisons (méfiance des canaux officiels, efficacité des nouvelles technologies...). On note également cette « soumission » qui prévaut depuis l'élan révolutionnaire de 2011 et qui contribue à la multiplication des dénonciations des maux de la société (corruption, cas de torture, abus de pouvoir, atteintes aux données personnelles...).

Pour bien élucider les traits caractéristiques de l'expérience tunisienne, nous examinerons simultanément la situation des lanceurs d'alerte en Tunisie, en droit, où ils apparaissent comme des "discrédités " réhabilités de la transition démocratique, avant de l'aborder dans les faits, où ils se montrent comme des "sentinelles" controversées de la transition démocratique, comme un "remède ", pour reprendre l'expression de Tocqueville, aux maux des démocraties modernes (corruption, terrorisme, violation de la vie privée...). 


\section{I- Les lanceurs d'alerte, décrédibilisés réhabilités de la transition démocratique en Tunisie}

11 Depuis quelques années nous assistons en Tunisie à une évolution mouvementée dans le statut des lanceurs d'alerte caractérisée par :

12 - la mise en place hésitante mais progressive d'un cadre juridique et institutionnel potentiellement favorable aux lanceurs d'alerte ;

13 - la mise en œuvre désordonnée mais salutaire d'initiatives étatiques et citoyennes relativement propices aux lanceurs d'alerte.

\section{A- La mise en place hésitante mais progressive d'un cadre juridique et institutionnel potentiellement favorable aux lanceurs d'alerte}

14 Le droit tunisien ne comporte pas actuellement de lois spécifiques dédiées à la protection des lanceurs d'alerte. Toutefois, certaines lois sectorielles offrent des dispositifs de protection qui s'appliquent à certaines catégories de personnes ou d'alertes dans des domaines délimités comme la lutte contre la corruption, la protection de l'enfance, la lutte contre le terrorisme et le blanchiment d'argent, la prévention de la torture ou la justice transitionnelle. Les travaux de recherche juridique en la matière sont très rares, contrairement à la doctrine actuellement très abondante en la matière dans le monde. La jurisprudence est très rare voire quasi inexistante.

15 Cet état du droit positif est appelé à évoluer pour divers facteurs.

\section{1- La consécration d'un nouveau socle constitutionnel valorisant les libertés et droits fondamentaux}

16 La nouvelle constitution tunisienne a innové et affirmé les jalons d'un système constitutionnel garant des droits et libertés, une sorte de "fond de garanties constitutionnelles révolutionnaires", qui bénéficieront assurément aux lanceurs d'alerte.

\section{1- L'innovation de la protection des libertés et droits fondamentaux dans la Nouvelle Constitution du 27 janvier 2014}

Cette innovation constitutionnelle a porté essentiellement sur les principes suivants :

- principe de lutte contre la corruption, la fraude et l'évasion fiscale : «L'État met en place les mécanismes propres à garantir le recouvrement des impôts et la lutte contre l'évasion et la fraude fiscales. L'État veille à la bonne gestion des deniers publics, prend les mesures nécessaires afin que leur dépense s'effectue selon les priorités de l'économie nationale et œuvre à contrecarrer la corruption (...) » (article 10).

19 - principe de la souveraineté du peuple sur les ressources naturelles: «Les ressources naturelles appartiennent au peuple tunisien. L'État exerce sa souveraineté sur ces ressources au nom du peuple (...) » (article 13).

20 - principe qui a « apporté de l'eau au moulin » des lanceurs d'alerte dans le domaine de l'énergie et qui a fait d'énormes échos à tel point que le ministère de l'Énergie et des 
Mines a procédé en juin 2016 à la publication des contrats pétroliers sur son site web dans la rubrique Open Data. Une première du genre ${ }^{7}$.

21 - principes d'intégrité et de bonne gouvernance : «l'administration publique est au service du citoyen et de l'intérêt général. Son organisation et son fonctionnement sont soumis aux principes de la neutralité, de l'égalité et de la continuité du service public, et conformément aux règles de la transparence, de l'intégrité, de l'efficacité, et de la responsabilité » (article 15).

22 - droit à l'information et droit d'accès à l'information: "L'État garantit le droit à l'information et le droit d'accès à l'information. L'État œuvre à garantir le droit d'accès aux réseaux de communication » (article 32).

23 - garantie des libertés académiques et liberté de la recherche scientifique: "Les libertés académiques et la liberté de la recherche scientifique sont garanties » (article 33).

24 - principe de liberté et d'indépendance de la profession d'avocat: «La profession d'avocat est libre et indépendante, elle participe à l'instauration de la justice et à la défense des droits et des libertés. L'avocat bénéficie des garanties légales le protégeant et lui permettant d'assurer ses fonctions" (article 105). Une spécificité constitutionnelle tunisienne.

25 - principe de l'indépendance de la justice : «Les magistrats sont nommés par décret présidentiel sur avis conforme du Conseil supérieur de la magistrature » (article 106).

26 - création de la Cour constitutionnelle (articles 118 à 124) : organe important pour le contrôle du respect des droits et libertés fondamentaux.

27 - création des instances constitutionnelles indépendantes: "Les instances constitutionnelles indépendantes œuvrent au renforcement de la démocratie. Toutes les institutions de l'État doivent faciliter l'accomplissement de leurs missions. Elles sont dotées de la personnalité juridique et de l'autonomie financière et administrative. Elles sont élues par l'Assemblée des Représentants du Peuple avec une majorité qualifiée » (article 125) : une autre particularité constitutionnelle tunisienne. Elles sont au nombre de cinq: l'Instance des élections; l'Instance de la communication audiovisuelle; l'Instance des droits de l'Homme ${ }^{8}$; l'Instance du développement durable et des droits des générations futures; l'Instance de la bonne gouvernance et de la lutte contre la corruption?.

\section{2- L'affermissement de la protection des libertés et droits fondamentaux dans la Nouvelle Constitution du 27 janvier 2014}

28 Plusieurs dispositions de la Nouvelle Constitution réaffirment la garantie des libertés et droits fondamentaux : libertés d'expression, d'information et de publication (article 31), liberté d'association (article $35 \mathrm{C}$.).

29 L'importance de cette consécration constitutionnelle apparait lorsque les lois sur les lanceurs d'alerte limitent la nature des dénonciations qui ouvrent droit à une protection ou restreignent la portée de celle-ci; les libertés et droits fondamentaux, comme la liberté d'expression, peuvent être fort utiles (hypothèse de la jurisprudence française "Clavaud $»^{10}$, cas du jugement rendu par le Tribunal administratif de Cergy Pontoise ${ }^{11}$. 
30 La garantie d'une protection constitutionnelle générale, effective et efficace, bénéficiant aux lanceurs d'alerte, dans le secteur public ou privé, dépendra certainement de l'interprétation que donnera la future Cour constitutionnelle à leurs libertés et droits fondamentaux prévus par la Nouvelle Constitution. La carence législative ou réglementaire dans la protection des lanceurs d'alerte serait compensée, dans ce cas, par un niveau élevé de protection des libertés et droits fondamentaux ${ }^{12}$.

31 Le mérite de cette conception qui appréhende la protection jurisprudentielle des lanceurs d'alerte à travers le perfectionnement de la protection générale des libertés et droits fondamentaux est de valoriser l'impact potentiel - et essentiel - des systèmes d'alerte éthique sur la remise en question, la régénérescence, et la consolidation d'une démocratie ${ }^{13}$.

\section{2- La réception des standards internationaux favorisant la bonne gouvernance et la lutte contre la corruption}

Depuis les premières heures de la transition démocratique en Tunisie, le besoin de la rupture avec l'injustice, l'oppression, la corruption se fait sentir et s'exprimer à tous les niveaux de l'État et de la société. L'un des moyens de réussir cette rupture a été, dans le cadre de la coopération internationale, d'engager toute une panoplie de réformes juridiques, institutionnelles et judicaires s'inspirant des bonnes pratiques et des lignes directrices universellement reconnues, notamment, dans les domaines des droits de l'Homme, de la justice, de la justice transitionnelle, de l'intégrité, de la bonne gouvernance et de la lutte contre la corruption.

\section{1- L'adoption des instruments internationaux conventionnels}

33 Le système juridique tunisien intègre diverses conventions internationales ayant trait à la protection des lanceurs d'alerte dont principalement :

34 - la Convention des Nations-Unies contre la corruption, approuvée par la Tunisie le 25 janvier $2008^{14}$ qui demande dans ses articles 8 et 33 aux États parties d'envisager des mesures de protection des dénonciateurs.

35 - la Convention arabe de lutte contre la corruption, signée par la Tunisie le 21 décembre 2010 qui stipule dans son article 14 : «Chaque État Partie fournit la protection légale nécessaire aux dénonciateurs, aux témoins, aux experts et aux victimes qui signalent des faits répréhensibles par cette convention; cette protection couvre leurs parents et les personnes qui leurs sont étroitement liés, de toute représailles ou intimidation potentielles (...)».

36 - la Convention africaine de l'Union africaine sur la prévention et la lutte contre la corruption, signée par la Tunisie le 27 janvier 2013 qui dispose dans son article 5 : « Aux fins de la réalisation des objectifs de la Convention, les États parties s'engagent à : (...) 5. Adopter des mesures législatives et autres pour protéger les informateurs et les témoins dans les cas de corruption et d'infractions assimilées, y compris leur identité,

6. Adopter des mesures afin de s'assurer que les citoyens signalent les cas de corruption, sans craindre éventuellement des représailles,

7. Adopter des mesures législatives nationales en vue de réprimer les auteurs de faux témoignages et de dénonciations calomnieuses contre des personnes innocentes dans les procès de corruption et infractions assimilées ». 


\section{2- L'adhésion aux standards internationaux non contraignants} législation tunisienne actuelle. Ni d'ailleurs leur régime juridique. Leur appréhension se fait souvent de manière sectorielle, parcellaire et sans réel fond commun de régulation. L'évocation de l'alerte et/ou du lanceur d'alerte se fait, la plupart du temps, dans l'optique de l'obligation de dénoncer certains faits délictueux, et non du droit de donner l'alerte.

\section{1- Les lois sectorielles traitant de l'alerte et des lanceurs d'alerte} institue une obligation de signalement incombant aux fonctionnaires publics : «toutes les autorités et tous les fonctionnaires sont tenus de dénoncer au procureur de la République les infractions qui sont parvenues à leur connaissance dans l'exercice de leurs fonctions et de lui transmettre tous les renseignements, les procès-verbaux et les actes qui y sont relatifs. En aucun cas, ils ne peuvent être actionnés en dénonciation calomnieuse ni en dommages-intérêts, en raison des avis qu'ils sont tenus de donner par le présent article, à moins d'établir leur mauvaise foi ».

Dans ses relations avec ses supérieurs hiérarchiques, le Décret institue, expressément, public au cas où « l'exécution d'instructions (...) constituent un délit ou un crime punis par la loi ». On pourrait y déceler une certaine reconnaissance de la "logique d'insoumission » qui structure la notion de déclenchement d'alerte, dans laquelle les ordres des supérieurs doivent en permanence se trouver questionnés. Le décret met à la charge de l'agent public, en revanche, une "obligation de divulgation interne » le 
contraignant à «informer son supérieur hiérarchique de toute violation de la loi rencontrée au travail ».

Dans ses relations avec ses collègues, l'agent public est tenu de « respecter l'intimité de ses collègues et de s'abstenir d'user d'informations concernant leur vie privée dans le but de leur nuire ». Resurgit alors le droit au respect de la vie privée. La limite entre faits relevant de la "vie privée » et faits relevant de «comportement répréhensible » est parfois, avouons-le, difficile à tracer.

Dans ses relations avec ses subordonnés, l'agent public est tenu de "s'engager à prodiguer ses ordres et instructions par écrit chaque fois qu'il reçoit une note écrite de la part de ses agents évoquant l'illégalité de ses ordres ou instructions ». Innovation du texte, devant une dénonciation écrite, la décision à prendre par le supérieur hiérarchique devrait parvenir, au dénonciateur, par écrit.

Dans ses relations avec les citoyens, l'agent public est tenu de « s'abstenir de divulguer les données et informations privées dont il a eu connaissance dans l'exercice de ses fonctions ou de les utiliser à des fins non professionnelles ", rappel de l'obligation de se conformer au secret professionnel. Or, la conciliation entre l'obligation du respect du secret professionnel et l'obligation de dénonciation des faits délictueux peut apparaître quelquefois difficile à cerner.

Dans ses relations avec les médias, le Décret met l'agent public dans une situation ambiguë et délicate face à la problématique de l'alerte éthique :

51 1. L'agent public ne peut fournir aucune déclaration, intervention, publication ou divulgation d'informations ou de documents officiels par l'intermédiaire de la presse ou autres médias, notamment des sujets se rapportant à l'exercice de ses fonctions ou à la structure publique dans laquelle il travaille, sans l'accord préalable et explicite de son supérieur hiérarchique ou du responsable de cette structure.

52 2. L'agent public est tenu de s'abstenir de toute déclaration, sous quelque forme que ce soit, qui irait à l'encontre de l'obligation de discrétion professionnelle et à l'encontre de l'intérêt suprême de l'État.

53 3. L'agent public ne doit pas procéder à la rétention d'informations ou de documents officiels qui pourraient ou devraient être rendus publics, ni diffuser des informations trompeuses ou inexactes sur des sujets se rapportant à ses fonctions ou à la structure publique dans laquelle il travaille.

54 4. L'agent public est tenu de ne communiquer des informations ou des documents en rapport avec ses fonctions ou la structure publique dans laquelle il travaille, que dans le respect des lois et règlements en vigueur ".

Rappel solennel d'une autre obligation déontologique, qui pèse sur l'agent public et qui pose, sans doute, le plus de difficultés : celle du devoir de discrétion professionnelle. Ce sont «les secrets de l'administration qui sont ici protégés »" Or, ce sont précisément ces «secrets" qui sont concernés lorsqu'un agent va signaler aux autorités compétentes les informations, renseignements et actes en rapport avec ses fonctions ou la structure publique dont il a eu connaissance dans, ou à l'occasion de, l'exercice de ses fonctions.

Bien qu'innovant, le Code de conduite et de déontologie de l'agent public promulgué par décret semble s'apprêter à une impérieuse harmonisation et modification pour mieux s'aligner sur les dispositions constitutionnelles et législatives en vigueur. Les 
multiples restrictions qu'il réaffirme avec insistance, affecteraient certainement, à notre avis, le sens, l'étendue et l'efficacité de tout dispositif d'alerte.

En l'état actuel, le droit de la fonction publique tunisien semble encore relativement réticent à la réception complète et efficace du mécanisme de l'alerte éthique en raison d'une pléiade d'obligations déontologiques (obligation d'obéissance hiérarchique, obligation de se conformer au secret professionnel, obligation de discrétion professionnelle...) difficile à concilier, du moins en apparence, avec la logique du déclenchement d'alerte.

Dans le domaine de la protection de l'enfance, le Code de protection de l'enfant, promulgué en 1995, institue un dispositif intéressant de signalement. Une obligation étendue d'alerte: "Toute personne, y compris celle qui est tenue au secret professionnel, est soumise au devoir de signaler au délégué à la Protection de l'Enfance tout ce qui est de nature à constituer une menace à la santé de l'enfant, ou à son intégrité physique ou morale (...) » (article 31); de même « toute personne majeure est tenue d'aider chaque enfant qui se présente à elle en vue d'informer le délégué à la Protection de l'Enfance ou de lui signaler l'existence d'une situation difficile qui menace l'enfant, ou l'un de ses frères, ou tout autre enfant » (article 32). Une protection contre les poursuites: «Nul ne peut être poursuivi devant les tribunaux pour avoir accompli de bonne foi le devoir de signalement» (article 33); et contre la divulgation d'identité : «il est interdit à toute personne de divulguer l'identité de celui qui s'est acquitté du devoir de signalement, sauf après son consentement ou dans les cas prévus par la loi » (article 34).

Dans le domaine du journalisme, le décret-loi n 2011-115 du 2 novembre 2011 relatif à la liberté de la presse, de l'imprimerie et de l'édition met clairement en exergue le droit à une information libre, pluraliste et transparente ainsi que le droit d'accès aux informations. L'article 9 prévoit ainsi qu'« il est interdit d'imposer des restrictions à la libre circulation des informations ou des restrictions pouvant entraver l'égalité des chances entre les différentes entreprises d'information dans l'obtention des informations, ou pouvant mettre en cause le droit du citoyen à une information libre, pluraliste et transparente ». L'article 10 dispose que « le journaliste, au même titre que tout citoyen, a un droit d'accès aux informations, nouvelles données et statistiques; il a le droit d'en obtenir communication auprès de leurs différentes sources selon les conditions, modalités et procédures prévues par le décret-loi nº 2011-41 du 26 mai 2011 relatif à l'accès aux documents administratifs des organismes publics, tel que modifié par le décret-loi $n^{\circ} 2011-54$ du 11 juin 2011. Le journaliste peut demander aux différentes sources précitées, toutes informations, nouvelles et statistiques en leur possession, à moins que ces matières ne soient couvertes par le secret en vertu de la loi ».

L'apport considérable en la matière réside incontestablement dans la protection reconnue aux sources externes du journaliste consacrée par l'article 11: "Sont protégées les sources du journaliste dans l'exercice de ses fonctions, ainsi que les sources de toute personne qui contribue à la confection de la matière journalistique. Il ne peut être procédé à la violation du secret de ces sources directement ou indirectement que pour un motif impérieux de sûreté de l'État ou de défense nationale et sous le contrôle de l'autorité juridictionnelle ${ }^{22}$. Est considérée comme violation du secret des sources, toutes enquêtes, tous actes de recherche et d'investigation, toutes écoutes de correspondances ou de communications, effectuées par l'autorité publique à 
l'encontre du journaliste pour découvrir ses sources ou à l'encontre de toute personne entretenant avec lui des relations particulières. Le journaliste ne peut faire l'objet d'aucune pression, de n'importe quelle autorité et il ne peut être également exigé d'un quelconque journaliste ou d'une quelconque personne participant à la confection de la matière journalistique de révéler ses sources d'information, sauf autorisation du juge judiciaire compétent et sous réserve que ces informations soient relatives à des infractions présentant un risque grave pour l'intégrité physique d'autrui, que leur divulgation soit nécessaire pour prévenir la commission de telles infractions et qu'elles soient du type d'informations ne pouvant être obtenues par tout autre moyen ». La violation de l'anonymat des "sources externes" du journaliste est pénalement sanctionnée.

\section{2- Une garantie encourageante dans le processus de protection des lanceurs d'alerte}

61 Dans le domaine de la lutte contre la corruption, le Décret-loi 2011-120 du 14 novembre 2011, relatif à la lutte contre la corruption pose les prémisses d'un régime protecteur des dénonciateurs de la corruption. L'article 11 stipule ainsi que «dans le cadre de sa politique de lutte contre la corruption, l'État garantit l'encouragement à la dénonciation des infractions de corruption, et ce, en diffusant la conscience sociale sur ses dangers, en diminuant les obstacles juridiques et pratiques empêchant la détection et la preuve de la corruption par des mesures de protection des victimes, des témoins et des dénonciateurs ». L'article 13 précise que «l'instance nationale de lutte contre la corruption (INLUCC), qui sera remplacée prochainement par l'Instance de la bonne gouvernance et de la lutte contre la corruption, est chargée notamment de recevoir les plaintes et dénonciations, procéder à l'instruction et la transmission des infractions de corruption aux autorités compétentes y compris la justice». L'article 15 dispose que « les services et organismes publics compétents sont tenus d'assister l'instance dans la collecte des informations et statistiques sur les questions entrant dans le cadre de ses missions et l'exécution des procédures de protection des victimes, témoins et dénonciateurs ».

Dans le domaine de la prévention de la torture, la loi organique n²013-43 du 23 octobre 2013, relative à l'instance nationale pour la prévention de la torture instaure un dispositif qui donne à l'Instance pouvoir de réception, d'investigation et de transmission des dénonciations, portant sur des «cas de torture et autres peines ou traitements cruels, inhumains ou dégradants dans les lieux de détention", provenant de «toute personne qui peut fournir des informations " aux autorités administratives ou judiciaires (articles 3 et 4). L'étendue de la protection des dénonciateurs se limite à l'immunité contre les poursuites: "Tout en respectant la législation relative à la protection des données personnelles, aucune personne ne peut être poursuivie pour avoir communiqué des renseignements ou avoir divulgué des secrets se rapportant à l'exercice de la torture ou informer son auteur » (article 14).

Dans le domaine de la justice transitionnelle, la loi organique n 2013-53 du 24 décembre 2013 relative à l'instauration de la justice transitionnelle et à son organisation institue un autre dispositif particulier. La loi appréhende "l'alerte » comme étant un «droit garanti» pour "tous les citoyens" et «toutes les générations successives de tunisiennes et de tunisiens " pour " la révélation de la vérité concernant les violations » des droits de l'Homme et « la préservation de la mémoire nationale » (articles 2 et 5). La loi charge l'Instance «Vérité et Dignité » de «faire des investigations sur les cas de 
disparition forcée non résolus, sur la base des communiqués et des plaintes qui lui seront présentés et déterminer le sort des victimes » et de " collecter les informations (...) en vue de constituer une base de données et d'élaborer un registre unifié des victimes de violations » (article 39). Pour accomplir ses missions, l'Instance jouit de larges prérogatives afin d'assurer la protection des dénonciateurs et dispose du pouvoir de prendre «toutes mesures appropriées en coopération avec les structures et les services compétents, pour protéger les témoins, les victimes, les experts et tous ceux qu'elle auditionne, quel que soit leur statut, au sujet des violations relevant des dispositions de la présente loi, et ce, en assurant les précautions sécuritaires, la protection contre l'incrimination et les agressions, et, la préservation de la confidentialité » (article 40).

64 La loi prévoit expressément une " obligation générale de divulgation » des faits et actes relevant de la compétence de l'Instance. Cette obligation pèse sur les personnes publiques: "les services de l'État, les organismes publics, les commissions, les collectivités locales, les entreprises et établissements publics, ainsi que l'ensemble des fonctionnaires publics sont tenus de fournir au président de l'instance, des déclarations comportant tous les faits dont ils ont eu connaissance ainsi que toutes les informations et les données qu'ils peuvent avoir recueillies dans l'exercice ou à l'occasion de l'exercice de leurs fonctions, et qui relèvent des attributions de l'instance ou qui sont susceptibles de l'aider à réaliser au mieux ses missions. Lesdits documents et données sont présentés directement à l'instance, à l'initiative des services et des parties précitées ou à la demande de l'Instance, le cas échéant " (article 51); elle concerne également les personnes privées: "toute personne physique ou morale est tenue de fournir à l'instance, tous les documents ou toutes les déclarations se rapportant aux faits dont elle a eu connaissance ou qu'elle a dû subir ainsi que toutes les informations qu'elle a pu recueillir et qui relèvent des attributions de l'instance. Un récépissé est remis à toute personne ayant fourni à l'instance, des documents, des plaintes ou des informations » (article 52).

La loi immunise les « dénonciateurs » contre toute éventuelle poursuite disciplinaire ou judiciaire en relation avec les missions de l'Instance : « le secret professionnel ne peut être opposé aux demandes de l'Instance pour obtenir les informations et les données, et ce, quelle que soit la nature et le statut de la personne physique ou morale en possession de celles-ci. Les dépositaires de ces documents confidentiels ne peuvent être sanctionnés pour les avoir divulgués à l'instance » (article 54).

Dans le domaine de la lutte contre le terrorisme, la loi organique n 2015-26 du 7 août 2015 , relative à la lutte contre le terrorisme et à la répression du blanchiment d'argent institue une obligation de dénonciation incombant à toute personne ayant eu connaissance de "faits, informations ou renseignements relatifs aux infractions terroristes ». L'article 37 de ladite loi, qui a suscité de très vifs débats, considère comme "accomplissant un crime terroriste", celui qui "refuse de signaler aux autorités compétentes les faits, informations ou renseignements relatifs aux infractions terroristes », et ce «nonobstant qu'il soit tenu au secret professionnel ». Sont exceptés de ces dispositions, les parents, les enfants et le conjoint ; sont également exceptés, les avocats, les médecins et les journalistes à l'occasion de l'exercice de leurs missions.

67 L'étendue de la protection de «toute personne » susceptible de donner l'alerte, dans ce cas, est très large. La loi prévoit, dans sa section 7 intitulée « Des mécanismes de la protection », la mise en œuvre de « toutes les mesures nécessaires ». 


\section{3- Le projet de loi relative à la dénonciation de la corruption et à la protection des dénonciateurs} informer le dénonciateur des suites, motivation du refus de la demande de la protection, recours juridictionnel contre les décisions relatives à la demande de la protection.

\section{l'identité, protection physique...) et légales (immunité contre les poursuites, charge de} la preuve renversée ou partagée; la dénonciation ne constitue pas une violation de secret professionnel...).

82 - Gratification financière pour les dénonciateurs : ce qui pourrait choquer certains esprits " puritains » voyant dans l'alerte un " geste civique »!

83 - Sanctions (pénales et/ou disciplinaires) : divulgation de l'identité du lanceur d'alerte, dénonciation de mauvaise foi.

L'Assemblée des Représentants des Peuples apportera certainement des amendements à ce projet de loi ambitieux. Les débats risquent d'être passionnants et effervescents. Une question primordiale s'impose, d'ores et déjà, concernant l'harmonisation entre ce 
projet de loi et le projet de loi, en cours d'élaboration également, relative à l'Instance indépendante constitutionnelle de bonne gouvernance et de lutte contre la corruption.

\section{B- La mise en œuvre désordonnée mais salutaire d'initiatives étatiques et citoyennes relativement propices aux lanceurs d'alerte}

Durant toute la période de transition, les pouvoirs publics tunisiens se sont attelés à reconstruire un État intègre, respectueux des droits de l'Homme et des exigences de la bonne gouvernance. L'œuvre n'est pas aisée; elle évolue, tant bien que mal, avec ses échecs et ses réussites, ses défis et ses opportunités. Signe du temps " révolutionnaire » ou "transitionnel», les organisations de la société civile n'ont pas manqué de participer activement à cette œuvre de reconstruction de l'État, de la société et de la démocratie.

\section{1- Des actions étatiques appuyant les lanceurs d'alerte}

Les actions en question sont innombrables : on s'en tiendra aux plus importantes.

\section{1- Le renforcement des structures et mécanismes facilitant la tache aux lanceurs d'alerte :}

87 - Création de l'Instance nationale de lutte contre la corruption (2011) : entérinant les recommandations émanant de la «Commission nationale d'investigation sur la corruption et la malversation $»^{25}$, une Instance nationale de lutte contre la corruption (INLUCC) a été mise en place pour enquêter sur les cas de corruption; cette dernière sera remplacée, au terme de la Nouvelle Constitution, par l'Instance de la bonne gouvernance et de la lutte contre la corruption.

88 - Création du Ministre auprès du chef du gouvernement chargé de la gouvernance et de la lutte contre la corruption (2011): Le ministère a été, notamment, chargé de coordonner les politiques de prévention de la corruption au sein de l'administration publique. Cette structure ministérielle a été supprimée en 2014; sa mission a été attribué à un secrétaire d'Etat auprès du chef du gouvernement, chargé de la gouvernance et de la fonction publique ${ }^{26}$. Ce poste de secrétaire d'Etat a été lui-même supprimé en 2015 ; la bonne gouvernance et la lutte contre la corruption ont été « reléguées » à une Direction générale de la Présidence du gouvernement. Le ministère a été finalement réinstauré.

89 - Création de l'Instance de la « Vérité et Dignité » (2013).

90 - Création de l'Instance nationale de la prévention contre la torture et autres peines ou traitement cruels, inhumains ou dégradants (2013).

91 - Création de l'Académie internationale de la bonne gouvernance de Tunis (2013) ${ }^{27}$ : la mission principale de l'Académie est de renforcer et développer les capacités des hauts cadres de l'administration, aux niveaux central, régional et local en conformité avec les principes de la bonne gouvernance.

92 - Création d'un portail de dénonciation d'actes de corruption en ligne: Cette plateforme électronique de participation populaire (e-people) permet la dénonciation électronique des actes de corruption et de la poursuite des responsables en justice ${ }^{28}$. Il faut reconnaitre néanmoins que le site dédié à la question auparavant (http:// 
www.anticorruption-idara.gov.tn) n'a pas réellement réalisé, semble-t-il, les effets escomptés. Lever les craintes de dénoncer des actes répréhensibles, qui pourraient impliquer des agents publics, sur un portail public, est un travail de longue haleine.

\section{2- L'adoption de programmes de coopération internationale améliorant l'appréhension des lanceurs d'alerte :}

93 - Projet «Approfondir les bases de l'intégrité en Tunisie» dans le cadre Programme MENA-OCDE pour la Gouvernance mettant en exergue quatre piliers : l'élaboration d'un code de conduite pour les agents publics, l'analyse du système de contrôle, d'audit et de gestion des risques, la protection des lanceurs d'alerte, et la transparence dans les marchés publics ${ }^{29}$.

94 - Projet « Appui à l'établissement d'un système national d'intégrité en Tunisie » dans le cadre Projet Régional du PNUD pour l'intégrité et la lutte contre la corruption dans les pays arabes (ACIAC) s'articulant autour de cinq axes: Développer une stratégie nationale et un plan d'action de lutte contre la corruption, Appuyer l'opérationnalisation de l'Instance Nationale de Lutte Contre la Corruption, Elaborer un diagnostic du cadre juridique de la lutte contre la corruption, Renforcer les capacités des acteurs et institutionnaliser les connaissances relatives à la lutte contre la corruption, Sensibiliser l'opinion publique sur l'intégrité et la lutte contre la corruption ${ }^{30}$.

95 - Projet «Scan d'Intégrité Tunisie » dans le cadre «Initiative CleanGovBiz » de l'OCDE reposant sur un diagnostic de treize dimensions (dont la protection des dénonciateurs d'abus) déterminantes pour la promotion d'une bonne gouvernance et pour la détection, la prévention de la corruption ainsi que les poursuites qui s'imposent ${ }^{31}$.

96 - Projet «Promotion de la bonne gouvernance, lutte contre la corruption et le blanchiment d'argent» dans le cadre du Programme de l'Union européenne et du Conseil de l'Europe «Renforcer la réforme démocratique dans les pays du voisinage méridional $»(\text { SNAC Tunisie) })^{32}$.

97 - Plan d'action national du «Partenariat pour un Gouvernement ouvert » 2014/2016 (Opengov). Ce plan d'action intervient dans le cadre de l'initiative du gouvernement d'adhérer au «Partenariat ouvert » avec la société civile et le secteur privé, qui vise à établir une bonne gouvernance, basée sur la transparence et impliquant les citoyens dans la mise en place des politiques publiques et dans la lutte contre la corruption à travers les technologies de communication et d'information ${ }^{33}$.

98 Tous les projets susmentionnés, ainsi que plusieurs autres en cours d'exécution ou d'élaboration, démontrent une dynamique favorable à la logique de l'alerte et animée par le souci d'appui à la gouvernance démocratique et à la redevabilité publique en Tunisie.

\section{2- Des actions citoyennes soutenant les lanceurs d'alerte}

99 Les actions émanant de la société civile sont nombreuses; on se référera aux plus édifiantes. 


\section{1- Les activités de diffusion et de sensibilisation : aperçu à titre illustratif}

100 - Forum national sur la dénonciation de la corruption et la protection des dénonciateurs, organisé par l'Association tunisienne de lutte contre la corruption (ATLUC) en avril 2013.

101 - Rapport sur le degré de conformité des lois tunisiennes avec la CNULCC soulignant l'impératif d'assurer la protection légale des dénonciateurs, élaboré par l'association « I watch » en décembre $2014^{34}$.

102 - Séminaire de renforcement de capacité, «Vers un système efficace pour la protection des dénonciateurs de corruption en Tunisie », organisé par le Programme MENA-OCDE pour la Gouvernance, le Programme des Nations-Unies pour le Développement (PNUD), en partenariat avec le Gouvernement tunisien, et en collaboration avec l'Instance Nationale de la Lutte Contre la Corruption (INLUCC) à Tunis, mars 2015.

103 - Journée d'échange sur le thème: «La dénonciation, outil de lutte contre la corruption» organisé par l'Instance Nationale de la Lutte Contre la Corruption (INLUCC) et l'association Tunisienne de la lutte contre la corruption (ATLUC), en partenariat avec le PNUD, en mars 2016.

104 - Cérémonial pour trois femmes-lanceuses d'alerte primées par l'organisation «I Watch » du prix du meilleur dénonciateur d'affaires de corruption au titre de l'année $2014^{35}$. Ces femmes ont divulgué des affaires qui pourraient être qualifiées de corruption, et ce, en s'adressant à la justice, aux médias et à travers les réseaux sociaux. Il s'agissait en l'occurrence d'une avocate, d'une jeune diplômée de l'enseignement supérieur et d'une journaliste.

\section{2- Les activités de clarification et d'accompagnement : aperçu à titre illustratif}

105 - Élaboration d'un Guide de Gouvernance et d'Intégrité pour les Entreprises Publiques Tunisiennes (2014) : La deuxième partie du Guide traite de la question de l'Intégrité dans les entreprises publiques et présente les outils de lutte contre la corruption suivants : le code de conduite, le devoir de Diligence et le «Whistleblowing ».

106 - Création de sites internet relativement sécurisés dédiés aux lancements d'alerte : www.billkamcha.com (créé par l'association «I watch » en 2013) ; https://nawaat.org/ portail/leaks (crée par le blog collectif indépendant « Nawaat.org »).

107 Tous les dispositifs de dénonciation prévus par le droit tunisien, précédemment décrits, méritent une évaluation de synthèse au plan de l'efficacité et au plan de l'équité.

$108 \mathrm{Au}$ plan de l'efficacité, le droit tunisien a marqué une nette amélioration dans la clarification du régime de l'alerte et de régulation de la protection des divulgateurs. La pratique suivra-t-elle le Droit, la question demeure posée dans les faits.

109 Au plan de l'équité, le Droit tunisien semble encore imbibé par une vision sectorielle et parcellaire des questions de l'alerte et des lanceurs d'alerte. La multiplication des dispositifs légaux de dénonciation (lutte contre la corruption, lutte contre le terrorisme, prévention de la torture, justice transitionnelle...) altère certainement l'accessibilité et la visibilité de la législation; et pose sérieusement le problème d'harmonisation et de coordination entre les différents textes et les différentes instances. La cohérence des différents systèmes instaurés est en jeu. À cela s'ajoute la délicate conciliation entre diverses exigences contradictoires: obligations 
déontologiques (obligation d'obéissance hiérarchique, obligation de se conformer au secret professionnel, obligation de discrétion professionnelle) et droit à la désobéissance ou libertés et droits fondamentaux (liberté d'expression, droit à l'information, liberté de la presse...).

110 Ce statut juridique des lanceurs d'alerte, encore imprécis et en pleine gestation, a affecté, à bien des égards, le processus de transition démocratique en Tunisie.

Entre instrumentalisation politique et médiatique et engagement éthique et civique, les lanceurs d'alerte essaient se frayer un chemin vers la démocratie.

\section{II- Les lanceurs d'alerte, sentinelles controversées de la transition démocratique en Tunisie}

112 La période de transition en Tunisie s'est caractérisée par un déferlement sans précédent de «fuites » et d'alertes touchant un large éventail de secteurs et émanant de diverses catégories de dénonciateurs. Les uns semblent animés par l'intérêt général ; les autres semblent mobilisés par des intérêts partisans voire néfastes pour une transition démocratique.

\section{A- L'instrumentalisation politique et médiatique des lanceurs d'alerte, facteur de déstabilisation de la transition démocratique}

La place des pratiques informelles dans les transitions démocratiques peut se révéler déterminante. Ne pouvant atteindre leurs objectifs politiques, économiques, sociaux ou professionnels à travers les institutions formelles, certains groupes "pro" ou « contre » révolution procèdent par des instruments extra-institutionnels, voire extralégaux. Les "fuites" de certaines informations ou document en Tunisie postrévolutionnaire semble s'inscrire dans le cadre de cette logique partisane.

\section{1- La manipulation politique néfaste des lanceurs d'alerte}

114 En principe, on évoque des « fuites » lorsque quelqu'un à l'intérieur d'une organisation communique à quelqu'un à l'extérieur des informations confidentielles ou non publiées. Cela fait référence souvent à des situations où l'on cherche à manipuler l'information diffusée par les médias dans des contextes bien déterminés. La fuite est organisée dans son propre intérêt et non motivée par l'intérêt général ${ }^{36}$.

La question s'est posée avec acuité dans le contexte transitionnel tunisien pour des considérations principalement politiques.

\section{1- Un déferlement des fuites attisant les tensions politiques et sociales}

La divulgation d'informations confidentielles ou non publiées via des canaux non officiels met l'accent sur les tensions politiques et sociales et les dangers qui hantent et qui guettent le processus de transition démocratique en Tunisie. Pour illustrer notre propos, citons quelques cas de fuites graves qui ont suscité un tollé général voire un « séisme politique »: 
117 - Divulgation d'enregistrement vidéo, tel le cas du fameux et sulfurant entretien de l'ancien ministre de l'intérieur, F. Errajhi, avec des journalistes en mai 2011.

118 - Divulgation de rapports encore confidentiels, tel le cas du rapport préliminaire de la Cour des comptes concernant la gestion financière et administrative de l'Instance supérieure indépendante pour les élections (ISIE), en décembre 2012.

119 - Divulgation de documents confidentiels, tel le cas des notes des services de la sécurité nationale relatifs à des assassinats politiques en septembre 2013 et février 2015.

120 Ce genre de fuites "orchestrées » ou "spontanées ", touchant des hommes politiques, des journalistes, des agents publics, demeure très controversé. Il soulève particulièrement la question problématique de la bonne ou de la mauvaise foi des divulgateurs.

121 Nous pourrons, à cet égard, adopter la position de certains auteurs : « Face au dégoût et à la répugnance que peut inspirer la dénonciation anonyme quand elle est chargée de venin, il faut des dispositifs (...) c'est-à-dire, d'une part, une institution qui puisse filtrer au tamis la qualité des informations révélées et, d'autre part, une loi qui réprime durement les lanceurs d'alerte qui, emplis de mauvaise foi, se draperont dans l'intérêt général pour mieux frapper les intérêts privés $»^{37}$.

Nous pensons cependant que la notion de «bonne foi " du divulgateur demeure discutée dès lors que la légitimité d'un déclenchement d'alerte ne tient pas tant à un élément subjectif - l'intention du lanceur d'alerte - qu'à un élément objectif, l'intérêt pour la société de la divulgation de l'information en question ${ }^{38}$.

\section{2- Un déferlement des fuites dangereux pour le processus démocratique}

123 Les cas des fuites relevés précédemment, ont eu souvent des effets néfastes sur la vie politique et risquaient de saper le processus démocratique en cours, en ravivant un climat de tension, de crise, de rupture et de violence: les clivages politiques et idéologiques s'attisent, les crises de gouvernements se succèdent, les tensions sociales s'exacerbent, les démissions ou révocations de responsables politiques ou administratifs se multiplient.

124 Certains argueront toutefois que ces fuites, apparemment néfastes pour le processus démocratique, peuvent amener, paradoxalement, les pouvoirs publics à faire preuve de plus de transparence, de communication et d'ouverture.

125 D'autres répliqueront, en revanche, que l'alerte interne formelle ne contribue pas nécessairement à l'intérêt général, parce qu'il est plus facile d'étouffer l'affaire et que cela peut masquer des défauts systémiques.

126 Le débat sérieux de la question ne semble pas prêt à être clos dans les démocraties ancrées ou naissantes ${ }^{39}$.

\section{2- La manipulation médiatique nuisible des lanceurs d'alerte}

127 Dans une transition démocratique, les médias aussi connaissent leur « métamorphose ». L'évolution d'une censure totale ou d'une autocensure à une liberté affirmée d'expression et d'information n'est pas aisée.

128 Le climat de liberté qui s'installa en Tunisie après l'élan révolutionnaire de 2011 , mettait cependant les médias devant des dilemmes complexes entre information et 
instrumentalisation, professionnalisme et populisme, neutralité et partialité, devoir d'information et exigence de sécurité nationale.

\section{1- Un traitement des fuites prêchant parfois par l'investissement émotionnel et politique}

Dans son rapport sur les libertés du 3 mai 2014, le Syndicat national des journalistes tunisiens expose un bilan peu réjouissant et y détaille les maux qui sapent la transition médiatique dont notamment l'instrumentalisation politique.

La médiatisation de certaines fuites, dont celles évoquées précédemment, a mis en lumière parfois une couverture médiatique inégale, voire partisane. Par manque de professionnalisme, par conquête d'audimat ou par instrumentalisation politique.

Nous croyons que cette instrumentalisation est due, en grande partie, au flou juridique qui entoure le signalement aux médias en Tunisie. La légifération en la matière et le ralliement aux standards internationaux, qui autorisent la divulgation aux médias en dernier ressort mais en respectant le principe de "gradation » dans l'utilisation des canaux d'alerte, semble de mise ${ }^{40}$.

\section{2- Un maniement impartial des fuites grave pour le processus démocratique}

Dans la fabrication du consentement: de la propagande médiatique en démocratie, Noam Chomsky et Edward Herman mettent en évidence une orientation quasisystématique de l'information diffusée par les médias dominants dans le sens des intérêts des pouvoirs politiques et financiers. En période de transition cette orientation est encore aggravée par la précarité du processus et l'instabilité des institutions.

Le clivage " pro » et "contre " révolution se trouvait souvent au cœur de certaines fuites dans les médias tunisiens. Des fuites qui engendraient parfois des réactions populaires violentes, des crises politiques graves et des contestations sociales persistantes.

134 En aucun cas, cependant, ce traitement impartial des fuites ne devrait affecter l'acquis fondamental de la transition démocratique en Tunisie: la liberté d'expression et d'information. Comme le note, à juste titre, Naji Bghouri, président du Syndicat national des journalistes tunisiens (SNJT), le non-respect, par les professionnels du secteur des critères de précision et d'investigation, en particulier dans un contexte sécuritaire tendu ne pourrait servir de prétexte pour tenter de restreindre la liberté d'information $^{41}$.

\section{B- L'engagement éthique et civique des lanceurs d'alerte, facteur de consolidation de la transition démocratique}

Dotés seulement de leur bonne intention, certains lanceurs d'alerte prennent des risques énormes en sortant de leur anonymat pour s'exposer à l'opinion publique et affronter des forces politiques et économiques, souvent, démesurément plus puissantes, influentes et protégées. Car, une fois l'alerte lancée et l'effet médiatique oublié, les auteurs de ces révélations se retrouvent seuls et commencent à tourner dans une spirale infernale de poursuites judiciaires, de menaces, de lynchages publics et de pressions psychologiques et économiques. En attestent les témoignages émouvant de 
certains lanceurs d'alerte qui ont décidé de privilégier l'intérêt général au détriment de tout intérêt personnel ${ }^{42}$.

William Bourdon excelle dans la description de cet héroïsme citoyen : « en ce début du XXIe siècle se dressent, de véritables héros modernes, des hommes qui, bravant parfois tous les risques, semblent vouloir privilégier l'intérêt général à l'exclusion de tout intérêt personnel. Ils sont les nouveaux grands désobéissants de notre époque. (..) Bien sûr, il existe des nuances dans les parcours de ces hommes mais un trait d'union les regroupe dans une même confrérie, celle des grands lanceurs d'alerte qui privilégient la défense de l'intérêt supérieur à celui qui semble devoir résulter du seul respect de la loi. Des hyper-citoyens. Des hommes prêts à révéler la face sombre des Etats ${ }^{43}$.

On démontrera l'importance de l'action des lanceurs d'alerte, comme acteurs vigilants de la démocratie naissante tunisienne dans le domaine de la justice transitionnelle, des droits de l'homme et de la lutte contre la corruption.

\section{1- Le rôle majeur des lanceurs d'alerte dans le processus de justice transitionnelle}

138 La justice transitionnelle, au sens de la loi tunisienne, est un processus intégré de mécanismes et de moyens mis en œuvre pour cerner les atteintes aux droits de l'Homme commises dans le passé et y remédier, et ce, en révélant la vérité, en demandant aux responsables de ces atteintes de rendre compte de leurs actes, en dédommageant les victimes et en rétablissant leur dignité afin de parvenir à la réconciliation nationale, préserver et archiver la mémoire collective, instaurer des garanties pour que ces atteintes ne se reproduisent plus, et permettre la transition d'une dictature à un régime démocratique contribuant à la consécration des droits de l'Homme.

139 Les lanceurs d'alerte apparaissent, dans ce contexte, comme un moyen incontournable de révélation de la vérité, un mécanisme efficace de la redevabilité et un outil nécessaire de la réforme institutionnelle.

\section{1- Un moyen de la révélation de la vérité}

140 On entend par révélation de la vérité, «l'ensemble des moyens, procédures et investigations adoptés pour le démantèlement du système de dictature, et ce par la détermination et l'identification de toutes les violations, la recherche de leurs causes, leurs circonstances, leurs origines, et les conditions dans lesquelles elles se sont produites ainsi que les résultats qui en découlent. Et en cas de décès, de disparition, de disparition forcée, connaître le sort et la localisation des victimes et l'identité des auteurs et responsables des actes qui en sont à l'origine ».

141 L'intervention des lanceurs d'alerte dans le processus de révélation de la vérité est sans aucun doute primordiale.

142 L'expérience tunisienne montre toutefois parfois des limites à cette intervention. En atteste l'affaire Feriani qui démontra la volonté de certains services de l'Etat de conserver des dossiers qui s'avéreraient compromettants au détriment de la vérité. L'affaire Samir Feriani a commencé après sa lettre ouverte, publiée sur les colonnes de l'Expert, dans laquelle il a pointé du doigt les agissements de certains hauts cadres de l'ancien régime. Cette alerte lui a coûté une comparution devant la justice militaire et une condamnation à une amende pour avoir imputé à un fonctionnaire public des faits 
illégaux en rapport avec ses fonctions, sans en établir la véracité. Si Feriani a été libéré grâce à une large mobilisation et une médiatisation conséquente, d'autres lanceurs d'alerte n'ont pas eu cette chance ${ }^{44}$. La vérité en souffrira certainement.

\section{2- Un mécanisme de la redevabilité et un outil de réforme des institutions}

La redevabilité et l'obligation de rendre compte désignent l'ensemble des mécanismes qui empêchent l'impunité ou la soustraction à la responsabilité. Parmi ces mécanismes, incontestablement, les lanceurs d'alerte prennent une place de taille.

La réforme des institutions vise à démanteler le système de corruption, de répression et de dictature, et à y remédier d'une manière à garantir que les violations ne se reproduisent plus, que soient respectés les droits de l'Homme et que soit consacré l'Etat de droit. Les lanceurs d'alerte constituent, dans ce sens, une force contre les velléités autoritaires, une pierre angulaire dans ce processus de démantèlement de la dictature et de constriction de la démocratie.

\section{2- Le rôle incontournable des lanceurs d'alerte dans la révélation des violations des droits de l'Homme}

En citant les mémoires d'Andreï Sakharov, le philosophe Thomas Hammarberg, souligne que «la menace d'une révélation freine les atteintes aux droits de l'Homme. Lorsque les signalements responsables sont protégés par la loi et par des mesures pratiques, on peut s'attendre à ce que les violations des droits de l'Homme diminuent » 45 .

Dans cet esprit, les lanceurs d'alerte contribuent en Tunisie à la prévention et à la limitation des atteintes aux droits de l'Homme. Des cas de torture ou de mauvais traitement ont été, par exemple, répertoriés et divulgués sur les réseaux sociaux pour alerter le public et les autorités. Le procédé a été souvent efficace. Mais la question de l'alerte informelle par des canaux non officiels reste toutefois pendante.

\section{3- Le rôle indispensable des lanceurs d'alerte dans la lutte contre la corruption}

147 En Tunisie, l'ampleur de la corruption a sans doute constitué l'une des causes les plus profondes de la Révolution du 14 janvier 2011. On assiste, depuis, à une forte propension de personnes et d'associations dénonçant certaines pratiques de corruption. On passe par tous les moyens de divulgation: structures publiques, parlement, médias, réseaux sociaux.

148 Ces dénonciations portaient souvent leurs fruits et aboutissaient à une certaine reconsidération des décisions prises ou des conventions conclues. On avancera comme exemple édifiant, les dénonciations des abus dans le secteur des hydrocarbures par la société civile dont notamment l'Association Tunisienne de Transparence des Mines et Energie (ATTEM), qui mettait en relief les défaillances liées à certains permis pétroliers. Ces actions ont conduit l'Assemblée Nationale Constituante a rejeté des projets de loi relatifs à la ratification d'avenants aux conventions qui régissent certains permis pétroliers ${ }^{46}$.

149 Se multipliaient aussi, sur les réseaux sociaux, les plateaux de télévision, dans les journaux, la révélation de cas de corruption dans le secteur public ou privé. L'alerte numérique et les cybers activistes se présentent de plus en plus, dans le contexte 
transitionnel tunisien, comme une arme redoutable contre les méfaits de la corruption avec, parfois, des dérives certainement à éviter. Il revient au législateur, d'en prendre acte et de légiférer.

\section{Conclusion}

\section{NOTES}

1. Razmik Panossian et Marc Lemieux, basé sur les notes d'Elizabeth Kellett et Yasmine Farret, «Entre révolution et répression: vers une transition démocratique au Moyen-Orient et en Afrique du Nord? ", Le Caire et Ottawa, le 2 Juin 2011, Table Ronde, rapport et recommandations. 2. Voir la définition récente du Conseil d'Etat français, «Le droit d'alerte: signaler, traiter, protéger », Étude adoptée le 25 février 2016 par l'assemblée générale plénière du Conseil d'Etat, La Documentation française, http://www.conseil-etat.fr/content/download/59086/527939/ version/1/file/2016\%20ce_etude_droit \%20d \%20alerte.pdf

3. Voir, https://nawaat.org/portail/2010/11/28/tunileaks-les-documents-devoiles-parwikileaks-concernant-la-tunisie-quelques-reactions-a-chaud.

4. Pris au sens de processus politique caractérisé par le passage progressif d'un régime de dictature à une démocratie, et sans polémiquer sur l'épistémologie des termes "diffusion démocratique ", " consolidations démocratique ", " construction démocratique ». Renée Fregosi insiste sur le fait que les changements de régime entre démocratie et dictature se produisent dans les deux sens, sans que l'on ne puisse jamais considérer aucune situation comme inéluctable ni irréversible. Elle pense que loin d'être des catégories étanches, les deux grandes manières de gérer l'ordre du pouvoir (autoritarisme et démocratie, imposition et libre choix) s'imbriquent et se mélangent bien souvent de façon complexe. Transitions à la démocratie, régressions 
autoritaires et régimes hybrides mettent en lumière cette complexité du politique, Parcours transatlantiques de la démocratie. Transition, consolidation, déstabilisation, Ed. Peter Lang, Bruxelles, 2011.

5. Selon l'expression consacrée par Kelly Piccard, « un contrepoids parmi les contre-pouvoirs : la figure de l'anonyme ", Communication pour le IXe Congrès national de l'Association Française du Droit Constitutionnel (AFDC), Lyon, 26, 27 et 28 juin 2014, http://www.droitconstitutionnel.org/ congresLyon/CommLD/D-picard_T2.pdf

6. Voir en ce sens, Eilan Kaplan, «The International emergence of legal protections for whistleblowers », The Journal of Public Inquiry, 2001, p. 37.

7. Voir, http://www.leconomistemaghrebin.com/2016/06/10/contrats-petroliers-rendus-publics

8. Actuellement : Haut Comité national des droits de l'homme et des libertés fondamentale.

9. Actuellement : Instance Nationale de Lutte Contre la Corruption (INLUCC).

10. Selon laquelle un employé de la société Dunlop a agi dans les limites de la liberté d'expression en donnant une interview à un journal, dans laquelle il critiquait les conditions de travail, Voir M. Thimert, « L'affaire Clavaud », L'Humanité, 3 mai 1995, http://www.humanite.fr/node/103516 11. Le jugement rendu par le Tribunal administratif de Cergy Pontoise le 15 juillet 2014 consacre le "principe général» de protection des "lanceurs d'alerte», Voir en ce sens, P. Cahez, «la liberté d'expression du lanceur d'alerte est un principe général du droit de la fonction publique ", Mediapart, 23 août 2014, https://blogs.mediapart.fr/patrick-cahez/blog/230814/laliberte-dexpression-du-lanceur-dalerte-est-un-principe-general-du-droit-de-la-fonctionpublique; J-Ph. Foegle, «Une première application paradoxale mais ambitieuse du régime de protection des fonctionnaires lanceurs d'alerte », La Revue des droits de l'homme [En ligne], Actualités Droits-Libertés, mis en ligne le 20 août 2014, consulté le 27 juin 2016. URL : http:// revdh.revues.org/863

12. Björn Fasterling et David Lewis, «Fuites, droit et liberté d'expression : la loi peut-elle promouvoir les dénonciations dans l'intérêt général ?", Revue internationale du Travail, vol. 153 (2014), $\mathrm{n}^{\circ} 1$, p. 81 et $\mathrm{s}$.

Voir sur la question en général, G. Devers, La protection du lanceur d'alerte par la jurisprudence, éd. Tim Buctu, 10 novembre 2015.

13. Voir sur la question en général, G. Devers, La protection du lanceur d'alerte par la jurisprudence, éd. Tim Buctu, 10 novembre 2015.

14. Loi $n^{\circ}$ 2008-16 du 25 Février 2008 portant approbation de la convention des Nations Unies contre la corruption; Décret $n^{\circ} 2008-762$ du 24 Mars 2008 portant ratification de la convention des Nations-Unies contre la corruption.

15. Voir par exemple, OCDE, Committing to Effective Whistleblower Protection, mars 2016, http://dx.doi.org/10.1787/9789264252639-en ; OCDE, Compendium of Best Practices and Guiding Principles, 2011, http://www.oecd.org/g20/topics/anti-corruption/48972967.pdf

16. Voir par exemple, UNODC, Groupe d'examen de l'application, Examen de l'application de la Convention des Nations-Unies contre la corruption, Résumé analytique : Tunisie, 26 mai 2015, https://www.unodc.org/documents/treaties/UNCAC/WorkingGroups/

ImplementationReviewGroup/1-5June2015/V1503711f.pdf

17. Voir par exemple, CE, Protection des lanceurs d'alerte, Recommandation CM/Rec (2014) 7 et exposé des motifs, https://www.coe.int/t/dghl/standardsetting/cdcj/ CDCJ \%20Recommendations/CMRec(2014)7F.pdf

18. Voir par exemple, TI, International, Principles for Whistleblower Legislation, http:// www.transparency.org/whatwedo/publication/

international_principles_for_whistleblower_legislation

19. Selon le juriste australien A.J Brown « le caractère complet d'une législation protectrice des lanceurs d'alerte, quel que soit le secteur couvert, dépend de trois critères : l'étendue des faits susceptibles d'être signalés; l'étendue et la diversité des secteurs d'activité couverts par la 
législation ; et, enfin, l'étendue des personnes pouvant bénéficier du processus et des protections prévues par la loi », Brown, A. J., « Towards « ideal » whistleblowing legislation? » Some lessons from recent Australian experience, E-Journal of International and Comparative Labour Studies (2013).

20. Sur la thématique de la désobéissance civile, Voir, D. Lochak, « Désobéir à la loi », in Pouvoirs et liberté. Etudes en l'honneur de Jacques Mourgeon, Bruylant, 1998, p. 98 ; R. Encinas de Munagorri, «La désobéissance civile: une source de droit?", RTD civ. 2005. 73; David Hiez, Bruno Villalba (dir.), La désobéissance civile: approches politique et juridique, Presses Universitaires du Septentrion, 2008.

21. S. Dyens, "La déontologie du fonctionnaire et l'alerte éthique sont-elles compatibles?», AJCT, 19 novembre 2012, p. 557.

22. La CEDH admet la limitation du secret des sources afin de prévenir des infractions particulièrement graves, si l'ingérence s'avère néanmoins proportionnée comme ce fut le cas lors d'une affaire dont elle a eu à connaître concernant un reportage d'un journaliste danois sur des réseaux pédophiles. Considérant qu'il s'agissait en l'espèce d'un impératif prépondérant d'intérêt général, la Cour a conclu à l'absence de violation de l'article 10 dans son arrêt Nordisk Film \& TV A/S c. Danemark du 8 décembre 2005.

23. http://www.economie.gouv.fr/adoption-du-projet-loi-sapin-2-en-premiere-lecture-a-lassemblee-nationale, consulté le 30 mars 2016.

24. Selon les standards internationaux et la jurisprudence de la Cour européenne des droits de l'homme - sauf cas spécifiques (implication de la hiérarchie dans le délit) -, l'alerte doit être effectuée d'abord en interne, puis auprès des autorités judiciaires ou administratives, ou la presse - si la gravité et l'urgence de l'alerte le justifient.

25. C'est une instance publique indépendante créée par le décret-loi $n^{\circ} 2011-7$ du 18 février 2011, JORT 22 février $2011 \mathrm{n}^{\circ} 13$ dont la mission s'est achevée après la publication de son rapport final. 26. Décret $n^{\circ} 2014-413$ du 3 février 2014, portant nomination des membres du gouvernement.

27. Décret $n^{\circ}$ 2013-3803 du 17 septembre 2013, portant création et organisation de l'académie internationale de la bonne gouvernance de Tunis.

28. http://www.pm.gov.tn/pm/actualites/actualite.php ?lang =fr\&id =8979

29. http://www.oecd.org/officialdocuments/publicdisplaydocumentpdf/ ?cote =GOV/MENA/ $\mathrm{A}(2015) 3 \&$ docLanguage $=\mathrm{Fr}$; http://www.oecd.org/officialdocuments/

publicdisplaydocumentpdf $/$ ?cote $=$ GOV $/$ MENA $/ \mathrm{A}(2015) 2 \&$ docLanguage $=\mathrm{Fr}$

30. http://www.tn.undp.org/content/tunisia/fr/home/operations/projects/ democratic_governance/appui-a-1_etablissement-dun-systeme-national-dintegrite-en-tunis.html 31. Les Scans d'Intégrité sont fondés sur la Boîte à Outils «CleanGovBiz» développée conjointement par l'OCDE, les Nations-Unies, la Banque mondiale, le Groupe d'Action financière, Transparency International et l'Initiative pour la transparence dans les industries extractives, dans le but d'aider les gouvernements qui souhaitent renforcer l'intégrité et combattre la corruption en coopération avec le secteur privé et la société civile. Voir, OCDE (2014), Renforcer l'intégrité en Tunisie : l'élaboration de normes pour les agents publics et le renforcement du système de déclaration de patrimoine, Éditions OCDE ; OCDE (2013), rapport de l'OCDE sur le cadre juridique de l'intégrité dans le secteur public en Tunisie, rapport de l'OCDE sur la gouvernance publique, Éditions OCDE, Paris.

32. https:// www.coe.int/t/dghl/cooperation/economiccrime/corruption/Projects/SNAC/pdf/ SNACTUN-Start-up-DOA_fr.pdf

33. http://www.ogptunisie.gov.tn

34. https://issuu.com/iwatch/docs/uncac_fran__ais

35. Voir, http://directinfo.webmanagercenter.com/2015/01/24/trois-denonciatrices-daffairesde-corruption-primees-par-lorganisation-i-watch, consulté le 06 avril 2015.

36. Björn Fasterling et David Lewis, article précité, p. 76. 
37. W. Bourdon, http://www.lemonde.fr/idees/article/2013/07/24/protegeons-mieux-leslanceurs-d-alerte_3453124_3232.html ; consulté le 27 mars 2015.

38. Au Royaume-Uni, c'est d'ailleurs pour cette raison que l'Enterprise and Regulatory Reform Act de 2013 a supprimé, au sein du «Public Interest Disclosure Act» de 1998, la condition de «bonne foi » du lanceur d'alerte, Emma Game, «Blowing the whistle : what are your rights? », in British Journal of Neuroscience Nursing, 2014, vol. 10, no 1, p. 46-47.

39. Voir en ce sens, S. Dyens, "Le lanceur d'alerte : et si on en débattait (enfin) sérieusement? ", Dalloz Actualité, 15/09/2015, http://www.dalloz-actualite.fr/chronique/lanceur-d-alerte-et-sien-debattait-enfin-serieusement.

40. Voir par exemple, le "Public Interest Disclosure Act » qui ouvre aux lanceurs d'alerte un nombre important et non limitatif de canaux de signalement, protégeant, en outre, les individus s'exprimant dans la presse dès lors que ceux-ci n'avaient, faute de réaction des pouvoirs publics, pas d'autres moyens pour faire aboutir leur signalement; la jurisprudence européenne, qui prévoit que la diffusion des informations s'opère "d'abord auprès de son supérieur ou d'une autre autorité ou instance compétente ", avant d'envisager la divulgation au public "en cas impossibilité manifeste d'agir autrement » (CEDH, 12 février 2008, GUJA c. Moldavie, § 142).

41. Voir, « Bghouri condamne l'instrumentalisation des médias », La Presse, 27/05/2016, http:// www.lapressenews.tn/article/bhgouri-condamne-linstrumentalisation-des-medias/94/5032

42. Voir par exemple, E. Cazi, « Frachon, Gibaud, Forissier...Sept lanceurs d'alerte détaillent leur vie d'après », Le Monde, 03/05/2016, http://mobile.lemonde.fr/societe/article/2016/05/03/ frachon-gibaud-forissier-sept-lanceurs-d-alerte-detaillent-leur-vie-d-apres_4912870_3224.html

43. W. Bourdon, article précité

44. Voir sur cette affaire, "libérer Samir Ferani », Nawaat, 15/07/2011, http://nawaat.org/ portail/2011/07/15/liberez-samir-feriani.

45. Paul Stephenson, Michael Levi, « La protection des donneurs d'alerte », Rapport d'étude sur la faisabilité d'un instrument juridique sur la protection des employés qui divulguent des informations dans l'intérêt public, Conseil de l'Europe, CDCJ(2012)9FIN, Strasbourg, décembre 2012, p. 40

46. Voir sur cette affaire, M. Hammami, «Leak: La Commission de l'Energie de l'ANC vient de rejeter un avenant à la convention du permis Amilcar avec British Gas », Nawaat, 05.06.2014, https://nawaat.org/portail/2014/06/05/leak-la-commission-de-lenergie-de-lanc-vient-de-

rejeter-un-avenant-a-la-convention-du-permis-amilcar-avec-british-gas/; H. Rebhi, «Tunisie British Gas: Amilcar, le permis qui fâche ", L'économiste maghrébin, 04.04.2014, http:// www.leconomistemaghrebin.com/2014/04/04/amilcar-permis-british-gas-fache.

\section{RÉSUMÉS}

Acteurs incontournables de la démocratie moderne, les lanceurs d'alertes "s'arrogent " et "protègent ", de plus en plus, la parole dans les pays en transition démocratique, au détriment de leurs intérêts personnels, pour dénoncer l'abus, l'oppression, l'injustice et la corruption. L'expérience tunisienne, berceau du «printemps arabe» en 2011, est, en la matière, riche d'enseignement. S'alignant sur le mouvement d'émergence internationale des législations protectrices des lanceurs d'alerte, leur statut en droit tunisien, malgré une certaine précarité et incomplétude persistante, connait une meilleure protection juridique et judiciaire. La «spécificité» de l'expérience ne chasse pas «l'universalité » des dilemmes posés par la 
problématique de l'alerte. Initiateurs de la révolte des esprits, de la « logique d'insoumission » et de la prise de la parole en Tunisie postrévolutionnaire, les lanceurs d'alertes évoluent entre reconnaissance et réhabilitation, parfois entre désapprobation et instrumentalisation. Fondamentalement, ils veillent à ce que la transition à la démocratie en Tunisie demeure irréversible.

Key players of modern democracy, the whistleblowers "arrogate" and "protect" increasingly, speech in countries in transition to democracy, to the detriment of their personal interests, to denounce abuse, oppression, injustice and corruption. The Tunisian experience, the cradle of the "Arab Spring" in 2011, is, in this area, rich in teaching. In line with the movement of international emerging legislation protecting whistleblowers, their status in Tunisian law, despite some persistent insecurity and incompleteness, knows better legal and judicial protection. "Specificity" of the experience does not hunt "universality" of the dilemmas posed by the problem of the whistleblowing. Initiators of the revolt of the spirits, of the "logic of insubordination" and of the public speaking in post-revolutionary Tunisia, the whistleblowers evolve between recognition and rehabilitation, sometimes between disapproval and instrumentalization. Basically, they ensure that the transition to democracy in Tunisia remains irreversible.

\section{INDEX}

Keywords : whistleblowers, democratic transitions, Tunisia, constitutional renewal, freedom of expression, sectoral legislation, draft specific law, fight against corruption, human rights, transitional justice, civic Initiatives, instrumentalisation, key actors of democratic process Mots-clés : lanceurs d'alerte, transitions démocratique, Tunisie, renouveau constitutionnel, liberté d'expression, lois sectorielles, projet de loi spécifique, lutte contre la corruption, droits de l'homme, justice transitionnelle, initiatives citoyennes, instrumentalisation, acteurs clés du processus démocratique

\section{AUTEUR}

\section{SOUHEIL KADDOUR}

Souheil Kaddour (1972, Tunisie) est enseignant-chercheur en droit public à l'université de Sousse depuis 2001. Il est membre de l'initiative académique anticorruption (ACAD/ONUDC) depuis 2015, et expert national anticorruption auprès du PNUD depuis 2016. De 2012 à 2015, il a occupé le poste de conseiller du ministre de la justice, des droits de l'Homme et de la justice transitionnelle. Il a publié plusieurs études et a participé à diverses conférences, nationales et internationales, dans les domaines du droit fiscal, de la gouvernance, de la lutte contre la corruption, des droits de l'Homme, et de la justice transitionnelle. 